\title{
MusicSpace: A Multi Perspective Browser for Music Albums
}

\author{
Hans Jörg Müller and Antonio Krüger \\ University of Münster, Münster, Germany
}

\begin{abstract}
Finding music albums in large databases is an inherently difficult problem, especially if users do not know exactly what they are looking for. MusicSpace lays out music albums spatially according to different perspectives and provides a Zoomable User Interface to enable navigation within these perspectives. The choice of perspectives was inspired by the theory of Conceptual Spaces, so similar items regarding each perspective are placed near to each other. We believe that the concept of laying out items according to Conceptual Spaces has the potential to generalize to all kind of real world items like pictures, food, wines or books that can be described by perceptual qualities as time, color, emotion or taste.
\end{abstract}

\section{Introduction}

Imagine a user entering her favorite music store. She does not know exactly what she is looking for, and the albums are laid out according to some schema. If the store is good, similar albums are placed near to each other, and if she understands how the albums are ordered, she can find albums she is looking for. But there are many dimensions the albums could be ordered by: genre, artist, release date, region, etc. When the collection is digitally available, e.g. as her personal MP3 collection or at an online music store, the spatial arrangement of the items could be adjusted according to her current search criteria. We propose to layout the albums according to Conceptual Spaces and suggest a navigation method within this representation with a Zoomable User Interface. This approach scales both to different display sizes, so the same interface can be used on a mobile MP3 player, a desktop PC or a wall-size display, as to different domains, like picture galleries, restaurants, wine stores, book stores etc.

\section{MusicSpace}

Conceptual Spaces [1] are a framework in the family of geometric models to describe conceptual knowledge using vector spaces. A music album can be identified with a point along multiple dimensions, like time, longitude and latitude of recording place, or the valence and arousal dimensions of associated emotions. 


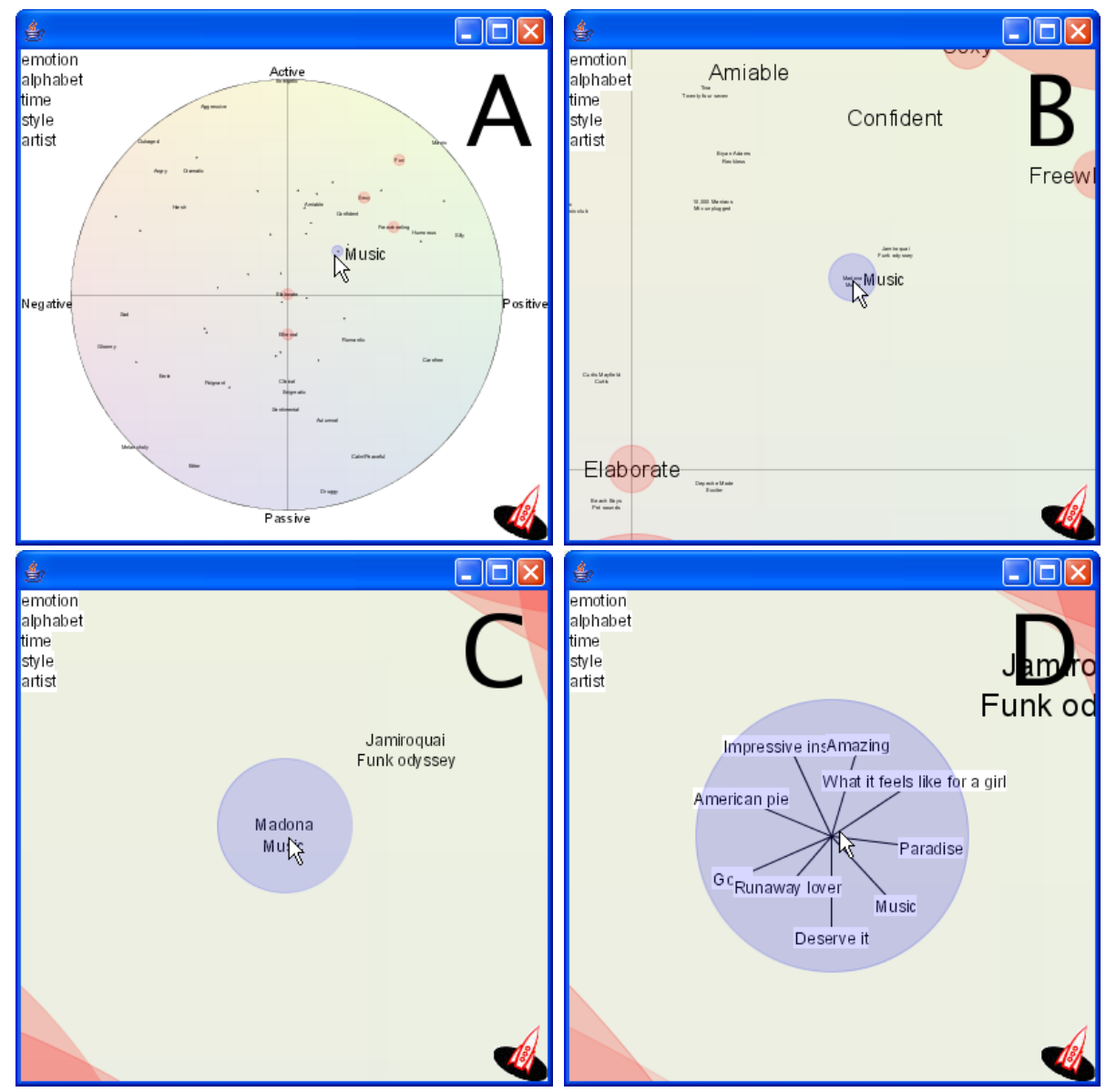

Fig. 1. Zooming to an album of Madonna in the emotion perspective (A, B, C), and playing songs from that album (D).

The two important aspects of this approach are that 1 . in each perspective, items similar regarding this perspective are placed near to each other, and 2 . if the user understands the structure of the perspective, she always knows where to look to find the items she is looking for. In the time domain, for example, the albums are ordered by their release date. Thus, the user can browse the space to find music albums that fit her search criteria, for example 'Music from the 80's'. To deal with the possibly infinite size and resolution of Conceptual Space domains and to solve the focus+context problem, we used a Zoomable User Interface. ZUIs allow for both infinite screen size and infinite resolution by providing continuous zooming in 2D. In the domain of emotion (Fig. 1), for example, an overview can be presented at a low zoom factor $(\mathrm{A})$, and the user can continuously zoom into a certain point to get more details $(B, C)$ and see the individual songs in the 


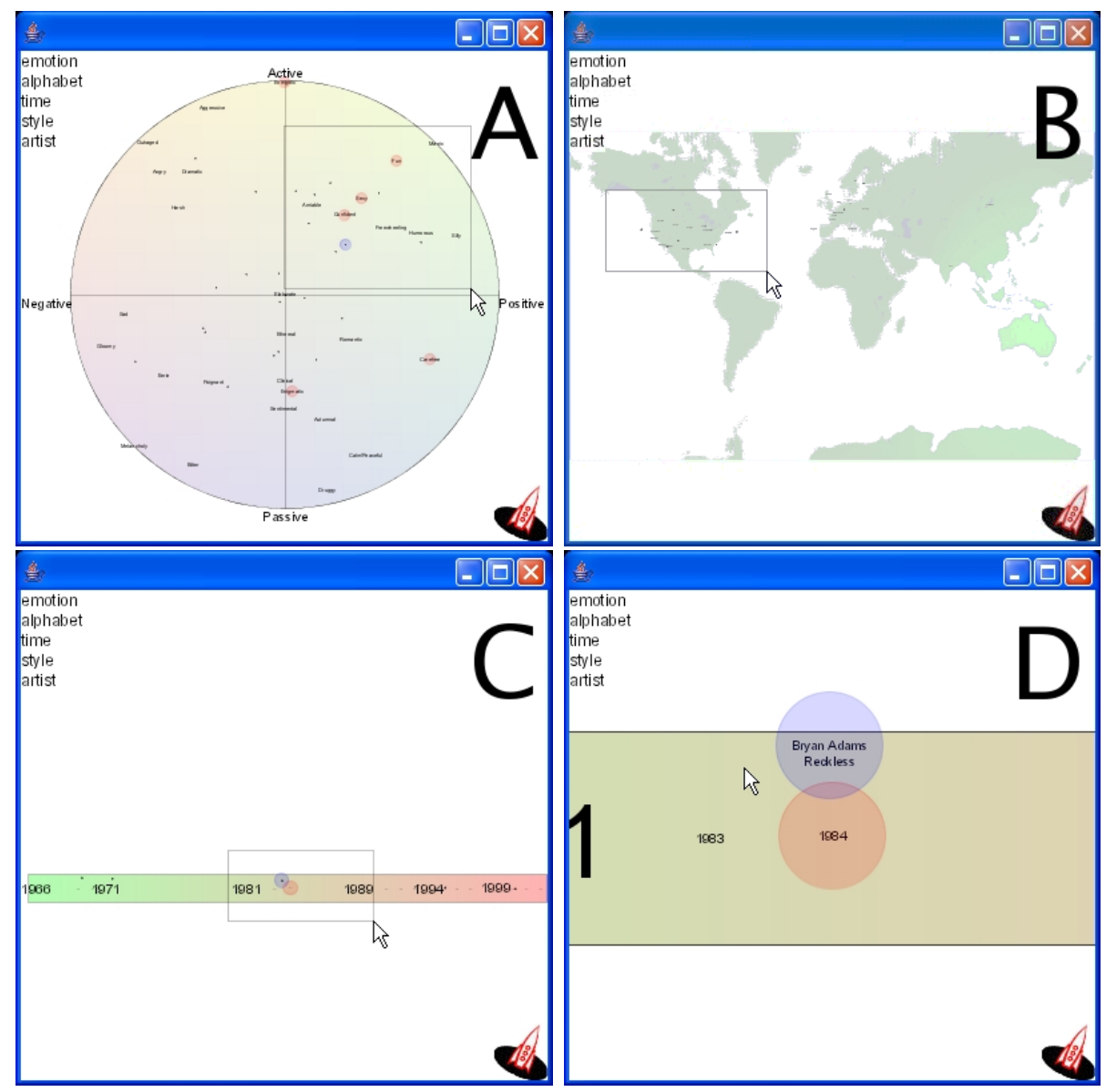

Fig. 2. Selecting some happy (A) american (B) music from the 80's (C,D).

album (D). The user can switch between perspectives by clicking on one of the buttons in the upper left corner. Furthermore, it is possible to apply dynamic query filters by selecting regions in multiple perspectives (Fig. 2). We believe that Conceptual Spaces provide a sophisticated conceptual framework for application designers to order items spatially and thus enable visual navigability using multiple perspectives.

Acknowledgements This work was done at the Tangible User Interface group of Sony Advanced Technology Center, Stuttgart.

\section{References}

1. Gärdenfors, P.: Conceptual Spaces: The geometry of thought. MIT Press, Cambridge, MA, 2000. 